ISSN 0103-9954

\title{
MODELAGEM DO CRESCIMENTO DE CLONES DE Eucalyptus USANDO O MODELO DE CHAPMAN-RICHARDS COM DIFERENTES DISTRIBUIÇÕES SIMÉTRICAS DOS ERROS
}

\author{
MODELING THE GROWTH OF Eucalyptus CLONES USING THE CHAPMAN-RICHARDS \\ MODEL WITH DIFFERENT SYMMETRICAL ERROR DISTRIBUTIONS
}

\author{
Luiz Medeiros de Araujo Lima Filho ${ }^{1}$ José Antônio Aleixo da Silva ${ }^{2}$ \\ Gauss Moutinho Cordeiro ${ }^{3}$ Rinaldo Luiz Caraciolo Ferreira ${ }^{4}$
}

\begin{abstract}
RESUMO
Objetivou-se neste trabalho estimar o crescimento em altura de clones de Eucalyptus usando o modelo de Chapman-Richards, considerando para os erros as distribuições: normal, t de Student ( $\mathrm{t}$ ) e Cauchy. Foram utilizados dados de clones de híbrido entre Eucalyptus urophylla x Eucalyptus tereticornis $x$ Eucalyptus pellita (polinização controlada), do Módulo de Experimentação Florestal para o Polo Gesseiro do Araripe, implantado em 2002. Utilizaram-se 83 árvores cujas alturas foram medidas durante seis anos e meio. Os parâmetros do modelo de Chapman-Richards foram obtidos maximizando a função de log-verossimilhança. Para comparação dos modelos foram utilizados os critérios de informação de Akaike (CIA) e Bayesiana (CIB) e o erro percentual absoluto médio (EPAM). O modelo usando a distribuição $t$ de Student com 2 graus de liberdade $\left(\mathrm{t}_{2}\right)$ obteve menores valores de CIA e CIB enquanto que o modelo de Cauchy obteve menor valor para o EPAM. Os resultados indicam que o modelo, considerando distribuição t para os erros, apresentou melhores estimativas do crescimento em altura de clones híbrido de Eucalyptus no Polo Gesseiro de Pernambuco.
\end{abstract}

Palavras-chave: máxima verossimilhança; modelos simétricos; distribuições robustas.

\begin{abstract}
The objective of this work was to estimate the height growth of Eucalyptus using the Chapman-Richards model considering for the errors the distributions normal, Student $\mathrm{t}(\mathrm{t})$ and Cauchy. The data set came from one hybrid clone of Eucalyptus urophylla x Eucalyptus tereticornis x E. pellita (controlled pollination), of the Forestry Experimental Module in the Gypsum Pole of Araripe, established in 2002. Eighty-three trees were used which heights were measured in all trees for six and half years. The parameters of the ChapmanRichards model were obtained maximizing the log-likelihood function. For comparison of the adjusted models were used the criteria of Akaike Information Criterion (AIC) and Bayesian (BIC) and the mean absolute percentage error (MAPE). The model using the $t$ distribution with 2 degrees of freedom $\left(t_{2}\right)$ had lower values of AIC and BIC and the model of Cauchy had lower value for MAPE. The results indicate that the model considering the $t$ distribution for the errors presented best estimates of height growth of Eucalyptus hybrid clones in Gypsum Pole of Pernambuco.
\end{abstract}

Keywords: maximum likelihood; symmetrical models; robust distributions.

1. Estatístico, Dr., Professor Adjunto do Departamento de Estatística, Universidade Federal da Paraíba, Cidade Universitária, CEP 58051-900, João Pessoa (PB). luiz@de.ufpb.br

2. Engenheiro Agrônomo, PhD., Professor Adjunto do Departamento de Ciência Florestal, Universidade Federal Rural de Pernambuco, Dois Irmãos, CEP 50740-540, Recife (PE). jaaleixo@uol.com.br

3. Estatístico, PhD., Professor Titular do Departamento de Estatística, Universidade Federal de Pernambuco, Cidade Universitária, CEP 50670-901, Recife (PE).gauss@de.ufpe.br

4. Engenheiro Florestal, Dr., Professor Adjunto do Departamento de Ciência Florestal, Universidade Federal Rural de Pernambuco, Dois Irmãos, CEP 50740-540, Recife (PE).rinaldof@dcfl.ufrpe.br

Recebido para publicação em 10/02/2010 e aceito em 12/07/2011 


\section{INTRODUÇÃO}

Historicamente, o gênero Eucalyptus é utilizado para os mais variados fins, tais como: lenha, carvão vegetal, celulose, dormentes ferroviários, postes para eletrificação, casca para curtir couro, óleos essenciais e construção civil (LIMA, 1993). Hoje, o Brasil se configura como um dos maiores produtores de Eucalyptus do mundo.

O Polo Gesseiro do Araripe, localizado na microrregião de Araripina, semiárido Pernambucano, é um grande consumidor de lenha que é usada na calcinação da gipsita. Essa microrregião abrange cinco municípios: Araripina, Bodocó, Ipubi, Ouricuri e Trindade, sendo responsável por cerca de $95 \%$ da produção de gesso industrializado no Brasil (ALBUQUERQUE, 2002).

O bioma caatinga, no qual está localizado o Polo Gesseiro do Araripe, vem sofrendo pressão, visto que é explorado de forma desordenada. Esse fato se deve, principalmente, a crescente demanda por recursos naturais renováveis, aumentando gradativamente a sua degradação com o incremento na produção de gesso.

A oferta de lenha na região proveniente dos planos de manejo sustentados não ultrapassa $4 \%$ da demanda da indústria do gesso. Sendo a lenha a principal fonte energética das calcinadoras, principalmente em função do baixo custo quando comparada com outras fontes energéticas, ocorre uma busca de lenha de outras regiões ou de lenha de desmatamentos ilegais.

Para atenuar esta situação, uma alternativa econômica e ambiental viável é o reflorestamento com florestas de rápido crescimento com destaque para o gênero Eucalyptus, por sua elevada taxa de crescimento, facilidade de reprodução, rusticidade e pelo altíssimo nível de melhoramento genético em produtividade e qualidade da madeira.

Em manejo florestal é imprescindível o conhecimento do crescimento da floresta. Em face deste fato, torna-se de interesse quantificar o crescimento e a produção de florestas, promovendo um planejamento criterioso da produção através da prescrição de regimes de manejos adequados visando a qualidade do produto final. Sendo assim, pode-se dizer que a predição do crescimento e da produção é parte fundamental do processo de planejamento dos povoamentos florestais.

As técnicas para prognosticar a dinâmica de um povoamento florestal são conhecidas como modelos de crescimento e produção (AVERY e
BURKHART,1994). Tais modelos são sínteses de observações e/ou fenômenos biológicos aplicados nas condições sob as quais foram coletados os dados. Na Engenharia Florestal, os modelos são imprescindíveis na prognose dos futuros recursos florestais, na escolha de metodologias silviculturais e apoio à tomada de decisões no manejo e na política florestal (SPATHELF e NUTTO, 2000).

A análise do crescimento em Ciências Florestais, utilizando modelos matemáticos e estatísticos, não é algo novo. Esse tipo de análise está cada vez mais sofisticada e, segundo Clutter et al. (1983) e Davis e Johnson (1987), uma das principais tarefas atribuídas ao profissional da área é entender como ocorre o crescimento das árvores de um povoamento, qual tratamento silvicultural adotar e prever qual será a época de corte e a produtividade do plantio.

O desenvolvimento de novos modelos de crescimento tem tido avanços consideráveis nas Ciências Florestais, como ocorreu com a generalização de Chapman-Richards para o modelo de Bertalanffy (1957) e trabalhos de Prodan (1968), dando um tratamento compreensivo de várias funções de crescimento e leis de crescimento estudadas com referência ao crescimento florestal. Esses modelos são formulações não lineares que, por meio de curvas de crescimento, procuram explicar as relações de crescimento inerentes a várias partes do organismo vegetal, até a sua maturidade, em situações ambientais distintas. Na literatura, são propostos vários modelos não lineares para descrever curvas de crescimento, tais como: logístico, Gompertz, Bertalanffy, monomolecular, Weibull, ChapmanRichards, Mitscherlich, Schumacher, Silva-Bailey, entre outros (BRITO et al., 2007).

O modelo normal não linear, por exemplo, que assume uma estrutura não linear para os parâmetros teve um grande avanço nos últimos anos. Esses modelos têm uma ampla variedade de aplicações, com a vantagem de obter parâmetros que são facilmente interpretáveis. Um modelo não linear bastante utilizado para descrever tais fenômenos em ciências florestais é o modelo de Chapman-Richards (SILVA, 1986).

Ao longo dos anos, modelos supondo erros normais vêm sendo utilizados para descrever a maioria dos fenômenos aleatórios, dado que a suposição de normalidade sempre é muito atrativa para os erros dos modelos de regressão com resposta contínua. Contudo, observa-se que as estimativas obtidas para os coeficientes dos modelos normais 
se mostram sensíveis à presença de observações extremas. Alternativas à suposição de erros normais têm sido propostas na literatura. Lange et al. (1989) propuseram o modelo baseado na suposição de erros t de Student. Taylor (1992) propôs o ajuste de um modelo de regressão linear supondo erro com distribuição exponencial potência com um parâmetro extra de forma. Galea et al. (2005) apresentaram alguns resultados sobre modelagem, em particular sobre o desenvolvimento da análise inferencial e de diagnóstico na classe de modelos não lineares com erros simétricos independentes. A fim de tentar reduzir a influência dessas observações extremas nas estimativas dos coeficientes, uma das alternativas é assumir para os erros distribuições com caudas mais pesadas do que a normal.

Considerando a grande utilidade no uso de modelos não lineares na análise do crescimento e produção de florestas e a real necessidade de considerar para os erros distribuições com caudas mais pesadas do que a normal, o objetivo deste trabalho consistiu em estimar crescimento em altura de clones híbridos de Eucalyptus usando modelos simétricos não lineares baseados em erros com distribuições mais robustas que a distribuição normal.

\section{MATERIAL E MÉTODO}

\section{Caracterização do experimento}

Em março de 2002 foi implantado na Estação Experimental do Instituto Agronômico de Pernambuco na Chapada do Araripe - PE o Módulo de Experimentação Florestal para o Polo Gesseiro do Araripe, que utilizou 15 clones do gênero Eucalyptus em uma área de 2,5 ha.

A região na qual o experimento foi realizado possui as seguintes coordenadas geográficas de posição: $07^{\circ} 29^{\prime} 00 \mathrm{~S}$ e $40^{\circ} 36^{\prime} 00 \mathrm{~W}$ e altitude de 816 metros. O clima regional predominante é do tipo BShwt de Koeppen, quente e seco de baixas latitudes, com chuvas de verão. A presença da Chapada do Araripe confere uma diferenciação no clima regional, elevando a pluviometria e registrando cerca de $735 \mathrm{~mm}$ anuais. A temperatura média é $24,6{ }^{\circ} \mathrm{C}$, a máxima é de $33^{\circ} \mathrm{C}$ e a mínima de $15,9^{\circ} \mathrm{C}$, com cerca de $2{ }^{\circ} \mathrm{C}$ de amplitude térmica. O solo é do tipo latossolo vermelho-amarelo (ENCARNAÇÃO, 1980; ARAUJO, 2004).

Neste trabalho a análise foi realizada para um dos clones referente ao tratamento híbrido de Eucalyptus urophylla $x$ Eucalyptus tereticornis $x$ Eucalyptus pellita (polinização controlada).
Foram utilizadas 83 árvores, sobreviventes das 100 árvores plantadas no início do experimento, sendo 25 árvores em cada uma das quatro repetições. A variável altura foi medida em todas as árvores ao longo do tempo, durante seis anos e meio, com a primeira medição sendo realizada aos dois meses, a segunda aos seis meses, e da segunda medição em diante com espaçamentos de seis meses. O tempo inicial considerado foi de dois meses que corresponde à idade em que as mudas foram plantadas no campo. As mudas dos clones de Eucalyptus foram provenientes da Comercial Agrícola Paineiras Ltda., localizada no município de Urbano Santos, no estado do Maranhão.

\section{Análise estatística}

A pressuposição de normalidade dos erros tem sido frequentemente considerada no ajuste desses modelos, sendo que, às vezes, o uso de distribuições simétricas mais robustas pode ser mais apropriado.

\section{Distribuições simétricas}

A família de distribuições simétricas gera uma classe geral de distribuições com a mesma simetria que a distribuição normal padrão. Entre essas distribuições, podemos citar: $\mathrm{t}$ e Cauchy. Maiores detalhes sobre a família de distribuições simétricas em modelos de regressão podem ser encontrados em Cysneiros e Paula (2005a).

Diz-se que a variável aleatória $Y$ tem distribuição simétrica, com suporte em $\mathbb{R}$, com parâmetros de locação $\mu \in \mathbb{R}$ e de dispersão $\phi>0$, se sua função de densidade de probabilidade for expressa por:

$$
f(y ; \mu, \phi)=\frac{1}{\sqrt{\pi}} \mathrm{g}(\mathrm{u}), \quad \mathrm{y} \in \mathbb{R}
$$

para alguma função $g(u)$, denominada função geradora de densidade, em que $u=\frac{(y-\mu)^{2}}{\phi}, \operatorname{com} g(u)>0$, para $u>0 \mathrm{e}$ $\int_{0}^{\infty} u^{-\frac{1}{2}} g(u) d u=1$. Esta condição é necessária para que $f(y ; u, \phi)$ seja uma função densidade de probabilidade. Assim, $Y \sim S(\mu, \phi)$ é denominada de variável aleatória simétrica.

É importante ressaltar que a família simétrica guarda a estrutura da distribuição normal, mas elimina a forma específica da densidade normal para incluir outras densidades simétricas.

A seguir, são apresentadas algumas distribuições simétricas com suporte na reta real. 


\section{Distribuição normal}

Diz-se que $Y \sim S(\mu, \phi)$ tem distribuição normal se sua função geradora de densidade $g(u)$ é da forma

$$
g(u)=\frac{1}{\sqrt{2 \phi}} \exp (-\mathrm{u} / 2), \quad \mathrm{u}>0,
$$

então, $Y$ tem distribuição normal denotada por $Y \sim N(\mu, \phi)$, (CYSNEIROS et al., 2005b).

\section{Distribuição $t$}

A distribuição $\mathrm{t}$ é utilizada para modelar o comportamento de dados que provêm de uma distribuição com caudas mais pesadas que a distribuição normal, permitindo reduzir a influência de observações aberrantes. Esta distribuição permite ajustar a curtose dos dados por meio dos graus de liberdade " $v$ ".

A variável aleatória $\quad Y \sim S(\mu, \phi)$ tem distribuição $\mathrm{t}$ se sua função densidade $g(u)$ é da forma

$$
g(u)=\frac{v^{v / 2}}{\mathrm{~B}(1 / 2, v / 2)}(v+u)^{-(v+1) / 2}, \quad \mathrm{u}>0, v>0,
$$

em que $B(1 / 2, v / 2)$ é a função beta. Assim, $Y$ é denotada por $Y \sim t(\mu, \phi, v)$, (CYSNEIROS et al., 2005b).

\section{Distribuição de Cauchy}

A variável aleatória $Y \sim S(\mu, \phi)$ tem distribuição de Cauchy se sua função geradora de densidade $g(u)$ é da forma

$$
g(u)=\frac{1}{\pi(1-u)}, \quad u>0 .
$$

Esta distribuição, denotada por $Y \sim C(\mu, \phi)$, é também conhecida como distribuição de Pearson Tipo VII (CYSNEIROS et al., 2005b). A distribuição de Cauchy também possui caudas mais pesadas que a distribuição normal.

Para uma melhor compreensão da forma das distribuições simétricas apresentadas, segue na Figura 1 a função de densidade da distribuição normal padrão, a função de densidade da distribuição $\mathrm{t}_{2}$ e a função de densidade da distribuição de Cauchy padrão.

\section{Modelos simétricos}

A família simétrica de densidades de locação-dispersão guarda a estrutura da distribuição normal, mas elimina a forma específica da densidade normal para incluir densidades simétricas com caudas mais leves ou mais pesadas do que as caudas da normal (CYSNEIROS et al., 2005b).

Para introduzir uma estrutura regressora na classe de distribuições (1), toma-se a componente sistemática do modelo linear generalizado para a média $\mu=E(Y)$ especificada por:

$$
g\left(\mu_{i}\right)=\eta_{i}(\beta)=h\left(x_{i}, \beta\right),
$$

em que $h(\cdot)$ é supostamente conhecida e duas vezes diferenciável, $\eta_{i}(\beta)$ é o preditor não linear, $x$ é uma matriz $n \times p$ de posto completo e $\beta=\left(\beta_{l}, \ldots, \beta_{p}\right)^{T}$ é um conjunto de parâmetros não lineares desconhecidos a serem estimados.

Os modelos simétricos assumem que as variáveis aleatórias $Y_{1}, \ldots, Y_{n}$ podem ser tratadas como distribuídas independentemente, seguindo a componente aleatória (1) e a componente sistemática (5). Desta forma, o modelo definido em (1) e (5) é dito modelo simétrico não linear.

\section{Inferência}

O principal objetivo na análise de modelos simétricos é fazer inferências no vetor de parâmetros $\beta$ e no parâmetro de dispersão $\phi$. A log-verossimilhança para os parâmetros do modelo pode ser expressa como:

$$
l(\beta, \phi)=-\frac{n}{2} \log \phi+\sum_{i=1}^{n} \log g\left\{\phi^{-1}\left(y_{i}-\mu_{i}\right)^{2}\right\} .
$$

A log-verossimilhança apresentada pode ser maximizada incondicionalmente usando alguns softwares como o SAS, Matlab, R ou a linguagem de programação Ox.

A função escore para o vetor de parâmetro $\beta$ e parâmetro de dispersão $\phi$ é expressa como:

$$
U_{\beta}=\phi^{-1} X^{T} H D(y-\mu)
$$

e

$$
U_{\phi}=(2 \phi)^{-1}\left\{\phi^{-1} Q(\mu, y)-n\right\} .
$$

Os parâmetros $\beta$ e $\phi$ são ortogonais. Assim, as estimativas de máxima verossimilhança $\hat{\beta}$ e $\hat{\phi}$ são assintoticamente independentes devido a sua normalidade assintótica e a estrutura bloco diagonal da matriz de informação conjunta, isto é, $K=\operatorname{diag}\left(K_{\beta}, k_{\phi}\right), \quad$ em que $K_{\beta}=4 a \phi^{-1} X^{T} H^{2} X \quad$ e $k_{\phi}=n(4 b-1) /\left(4 \phi^{2}\right)$ são, respectivamente, as matrizes de informação para $\beta$ e $\phi$. 


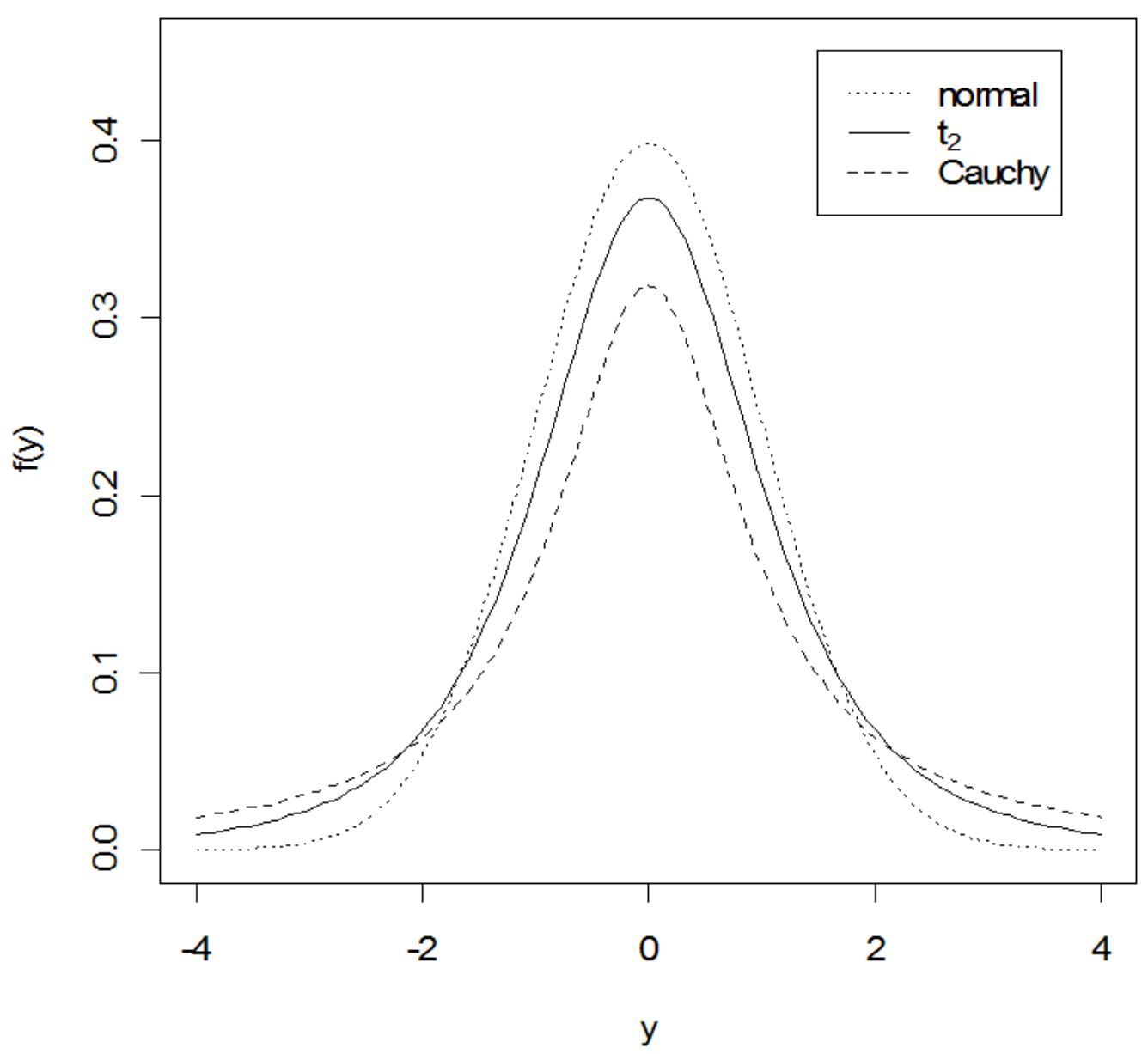

FIGURA 1: Densidades das distribuições normal padrão, $\mathrm{t}_{2}$ e Cauchy padrão.

FIGURE 1: Densities of the standard normal distribution, $\mathrm{t}_{2}$ and standard Cauchy.

Os estimadores de máxima verossimilhança de $\beta$ e $\phi$ não podem ser resolvidos explicitamente. No entanto, podem ser resolvidos por meio de um método iterativo, como por exemplo, o método escore de Fisher. Assim, o processo iterativo se reduz a

$$
\beta^{(m+1)}=\left(X^{T} H^{(m) 2} X\right)^{-1} X^{T} H^{(m) 2} \delta^{m}
$$

$\mathrm{e}$

$$
\phi^{(m+1)}=\frac{1}{n} Q\left(\mu^{(m)}, y\right)
$$

em que

$$
\delta^{(m)}=\eta^{m}+(4 a)^{-1} H^{(m)(-1)} D^{(m)}\left(y-\mu^{(m)}\right) .
$$

Aproximações iniciais $\beta^{(I)}$ e $\phi^{(I)}$ escolhidas são usadas para avaliar $H^{(l)}, D^{(I)}$ e $\delta^{(I)}$ e assim produzirem a próxima estimativa para $\beta^{(2)}$. Então, atualiza-se $\mu^{(2)}$ e $Q\left(\mu^{(2)}, y\right)$ para encontrar $\phi^{(2)}$ e assim continuando as iterações até que as estimativas $\beta \mathrm{e}$ $\phi$ sejam obtidas.

As expressões de $D, a$ e $b$ são facilmente deduzidas para muitas distribuições simétricas e podem ser encontradas na literatura especializada (CYSNEIROS, 2004).

\section{Modelo de Chapman-Richards}

Para estimar a altura das árvores foi utilizado o modelo de Chapman-Richards. Os modelos foram ajustados supondo diferentes distribuições simétricas para os erros. O procedimento de ajuste do modelo consistiu em estimar os parâmetros do modelo de Chapman-Richards maximizando a log-verossimilhança da distribuição simétrica adotada (2, 3 e 4). Dessa forma, foi possível ajustar o modelo adotado para qualquer distribuição da família simétrica. Este procedimento foi realizado por meio da Proc NLP do SAS. Para esse procedimento foi necessária apenas a utilização da log-verossimilhança para uma única observação. 
O modelo de Chapman-Richards na estrutura de modelos lineares generalizados para a média $\mu=E(Y)$ é expresso por:

$$
\mu=U\{1-\exp (-k T)\}^{\theta}
$$

em que $U$ é o termo assintótico' que significa o último nível de crescimento, $k$ é a taxa de crescimento, $\theta$ é o parâmetro de forma e $T$ corresponde à idade da árvore em meses. As estimativas dos parâmetros do modelo de Chapman-Richards serão apresentadas em conjunto com os seus respectivos erros padrão.

O próximo passo foi definir a logverossimilhança para as distribuições simétricas adotadas. A log-verossimilhança para o caso geral é dada por:

$$
l(\beta, \phi)=-\frac{1}{2} \log (\phi)-\log (\mathrm{g}(\mathrm{u})) .
$$

em que $u=\frac{(y-\mu)^{2}}{\phi}, \phi$ o parâmetro de escala e $\mu$ o parâmetro de locação.

Com os valores iniciais dos parâmetros do modelo de Chapman-Richards e de $\phi$ aplicou-se o Proc NLP para estimar os parâmetros de forma a maximizar a log-verossimilhança.

\section{Critérios de seleção}

Para comparar os modelos ajustados aos dados utilizou-se o critério de informação de Akaike (CIA) (AKAIKE, 1974), critério de informação Bayesiana (CIB) (AKAIKE, 1978) e o erro percentual absoluto médio (EPAM).

O critério de informação de Akaike é definido por:

$$
C I A=-2 \hat{\imath}+2 r,
$$

em que $\hat{l}$ é a log-verossimilhança maximizada e $r=p+1 ; p$ representa o número de parâmetros do modelo. A equação com o menor valor do CIA pode ser considerada como a que melhor explica a variabilidade dos dados.

O critério de informação Bayesiana é expresso como:

$$
C I B=-2 \hat{l}+r \log (n),
$$

em que $n$ é o número de observações. O modelo com o menor valor do CIB pode ser considerado como o que melhor explica a variabilidade dos dados.

Para comparar os modelos foi utilizado também o erro percentual absoluto médio EPAM, expresso por:

$$
E P A M=\frac{100 \%}{n} \sum_{i=1}^{n} \frac{\left|y_{i}-\widehat{y}_{i}\right|}{y_{i}},
$$

em que $y_{i}$ são os valores observados, $\hat{y}_{i}$ são os valores ajustados e $n$ é o tamanho da amostra. $\mathrm{O}$ modelo que tiver menor valor do EPAM é o que melhor explica os dados.

Para realização deste trabalho foram utilizados os softwares R e SAS. O software R foi utilizado para gerar gráficos e calcular algumas estatísticas apresentadas. O SAS foi utilizado para ajustar o modelo de Chapman-Richards para as distribuições simétricas adotadas.

\section{RESULTADOS E DISCUSSÃO}

Inicialmente, foi realizada uma análise descritiva dos dados com o intuito de compreender melhor o comportamento da variável em estudo. Observam-se na Tabela 1 baixos valores para os coeficientes de variação. A altura média das árvores na primeira medição, aos dois meses, foi de $0,4308 \mathrm{~m}$. Na última medição, realizada aos 78 meses, observou-se uma altura média de $14,966 \mathrm{~m}$.

As estimativas dos parâmetros dos modelos simétricos, bem como do parâmetro de dispersão $\phi$ são encontradas na Tabela 2. Observam-se valores próximos para as estimativas dos parâmetros $U, k$ e $\theta$ para os três modelos considerados. No entanto, o mesmo comportamento não foi observado para o parâmetro de dispersão $\phi$. Observa-se ainda que o modelo normal proporcionou a maior estimativa para o parâmetro $U$ que representa o último nível de crescimento.

Para escolher o modelo que melhor se ajustou aos dados são apresentados na Tabela 3 os critérios CIA, CIB e EPAM. $\mathrm{O}_{2}$ foi o modelo que apresentou o menor CIA $(-4,4732)$ e CIB $(-3,7322)$, sendo assim a equação mais indicada para representar os dados de acordo com esses critérios. De acordo com o EPAM, o modelo de Cauchy apresentou o menor valor $(9,1625)$, enquanto $\mathrm{o}$ modelo $t_{2}$ apresentou um valor bem próximo $(9,1782)$. Os resultados encontrados são bastante promissores, uma vez que o EPAM teve uma redução quando adotados os modelos $t_{2}$ ou Cauchy em relação ao modelo normal geralmente adotado em Ciências Florestais.

Observam-se na Figura 2 as curvas de 
TABELA 1: Idade em meses, média, desvio padrão (D.P.) e coeficiente de variação (C.V.) da altura do clone híbrido entre Eucalyptus urophylla x Eucalyptus tereticornis x Eucalyptus pellita.

TABLE 1: Age in months, mean, standard deviation (S.D.) and coefficient of variation (C.V.) of the height of the hybrid clone of Eucalyptus urophylla $\mathrm{x}$ Eucalyptus tereticornis x Eucalyptus pellita.

\begin{tabular}{cccc}
\hline T (idade em meses) & Média & D.P. & C.V. \\
\hline 2 & 0,4308 & 0,0340 & 7,8954 \\
6 & 1,2011 & 0,0672 & 5,5937 \\
12 & 3,9855 & 0,3094 & 7,7634 \\
18 & 6,0204 & 0,5868 & 9,7468 \\
24 & 8,4405 & 0,5820 & 6,8959 \\
30 & 9,7553 & 0,8393 & 8,6039 \\
36 & 10,5718 & 0,8850 & 8,3715 \\
42 & 11,1614 & 0,9027 & 8,0874 \\
48 & 11,6985 & 0,9108 & 7,7856 \\
54 & 12,2620 & 1,0080 & 8,2205 \\
60 & 12,8648 & 0,9945 & 7,7302 \\
66 & 13,4244 & 0,9922 & 7,3913 \\
72 & 14,1362 & 0,9725 & 6,8793 \\
78 & 14,9660 & 0,9757 & 6,5196 \\
\hline
\end{tabular}

TABELA 2: Estimativas e erros padrão para os parâmetros dos modelos simétricos.

TABLE 2: Estimates and standard errors of the parameters of symmetrical models.

\begin{tabular}{cccc}
\hline Distribuições & Parâmetros & Estimativa & Erro Padrão \\
\hline \multirow{4}{*}{ Normal } & $U$ & 15,009 & 0,554 \\
& $k$ & 0,041 & 0,006 \\
& $\theta$ & 1,397 & 0,177 \\
& $\phi$ & 0,645 & 0,121 \\
\hline \multirow{2}{*}{$\mathrm{t}_{2}$} & $U$ & 14,572 & 0,522 \\
& $k$ & 0,044 & 0,006 \\
& $\theta$ & 1,471 & 0,174 \\
\multirow{3}{*}{ Cauchy } & $\phi$ & 0,335 & 0,096 \\
& $U$ & 14,425 & 0,569 \\
& $k$ & 0,044 & 0,006 \\
& $\theta$ & 1,466 & 0,172 \\
\hline
\end{tabular}

TABELA 3: Critérios de seleção dos modelos.

TABLE 3: Selection criteria of models.

\begin{tabular}{cccc}
\hline Distribuições & CIA & CIB & EPAM \\
\hline Normal & 39,4198 & 40,1607 & 9,5224 \\
$\mathrm{t}_{2}$ & $-4,4732$ & $-3,7322$ & 9,1782 \\
Cauchy & 33,1796 & 33,9205 & 9,1625 \\
\hline
\end{tabular}

crescimento para cada uma das quatro repetições, bem como a curva estimada através do modelo $t_{2}$.

Na Figura 3 é apresentado o gráfico dos valores observados versus valores ajustados para o modelo $t_{2}$. Observa-se que não há indícios de afastamento da suposição de que o modelo $t_{2}$ é adequado para os dados.

Alguns estudos revelaram que a distribuição $t$ foi a mais adequada em modelagem na área de Ciências Florestais. Santos (2010) utilizou modelos simétricos transformados na estimativa volumétrica em híbrido de Eucalyptus tereticornis. No trabalho citado, observou-se que o modelo não linear de Schumacher e Hall combinado com a distribuição $t_{2}$ ajustou-se melhor aos dados. Em 2010, Barros abordou os modelos simétricos transformados através da inferência Bayesiana via algoritmo de Metropolis-Hastings. Os resultados obtidos indicaram o modelo $t_{2}$ via inferência Bayesiana como o modelo que melhor se ajustou aos dados de crescimento em altura de Eucalyptus urophylla.

\section{CONCLUSÕES}

O modelo $t_{2}$ obteve os menores valores segundo os critérios CIA e CIB, bem como um valor baixo para o EPAM, próximo do valor obtido para modelo Cauchy, que foi o menor valor encontrado. Os resultados encontrados são bastante animadores, uma vez que o erro percentual absoluto médio teve uma redução de $3,6 \%$ quando adotado o modelo $t_{2}$ em relação ao modelo normal. Assim, conclui-se que o modelo $\mathrm{t}^{2}$ foi o mais apropriado para estimar crescimento em altura de clones híbridos de Eucalyptus.

Dessa forma, os modelos simétricos são bastante relevantes para os estudos de modelos de crescimento de forma prática e bastante úteis para análise de dados reais, contribuindo assim de forma efetiva, no sentido de ampliar as possibilidades de análise para os modelos de crescimento adotados em Ciências Florestais. 


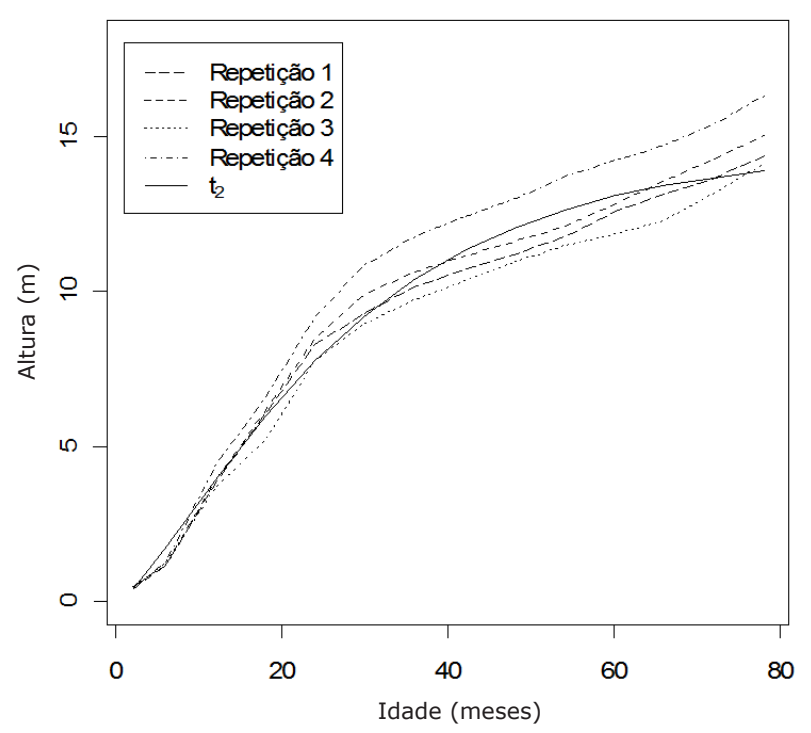

FIGURA 2: Gráfico de linha das repetições ao longo do tempo.

FIGURE 2: Line graph of repetitions along the time.

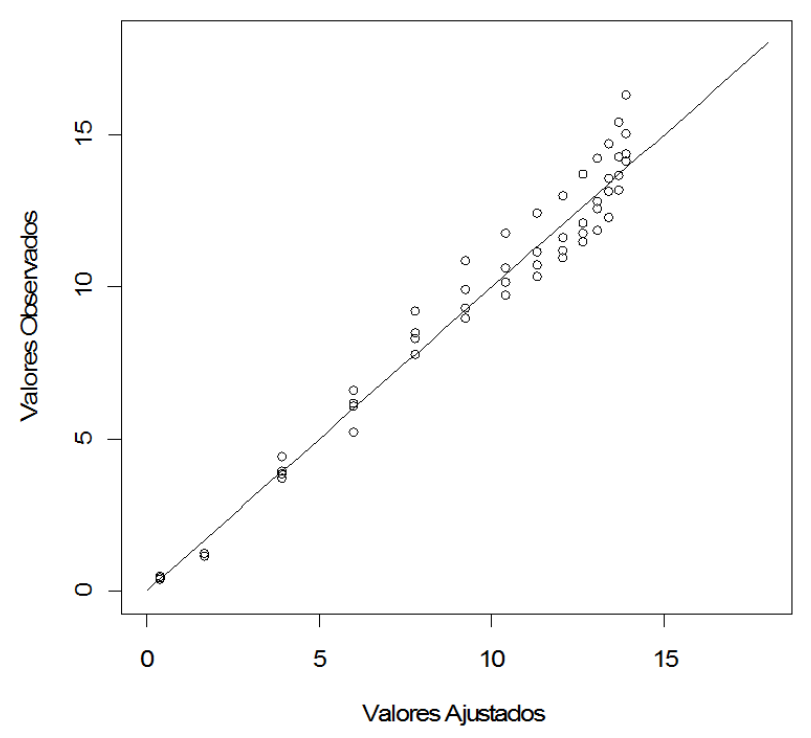

FIGURA 3: Valores observados versus valores ajustados para o modelo $t_{2}$.

FIGURE 3: Observed values versus fitted values for model $t_{2}$.

\section{REFERÊNCIAS BIBLIOGRÁFICAS}

AKAIKE, H. A new look at the Bayes procedure. Biometrika, London, v. 65, n. 1, p. 53-59, 1978.

AKAIKE, H. A new look at the statistical model identification. IEEE Transactions on Automatic Control, Boston,v. 19, n. 6, p. 716-723, 1974.

ALBUQUERQUE, J. L. Diagnóstico ambiental e questões estratégicas: Uma análise considerando o Pólo Gesseiro do Sertão do Araripe - Estado de Pernambuco. 2002. 185 f. Tese (Doutorado em Ciência Florestal) - Universidade Federal do Paraná, Curitiba. 2002.

ARAuJO, S. M. S. O Pólo Gesseiro do Araripe: unidades geo-ambientais e impactos da mineração. 2004. 276 f. Tese (Doutorado em Geociências) Universidade Estadual de Campinas, Campinas, 2004.

AVERY, T. E.; BURKHART, H. Forest management. 4th ed. New York: McGraw-Hill, 1994. $432 \mathrm{p}$.

BARROS, K. N. N. O. Abordagem clássica e Bayesiana em modelos simétricos transformados aplicados à estimativa de crescimento em altura de Eucalyptus urophylla no Polo Gesseiro do Araripe-PE. 2010. 84 f. Dissertação (Mestrado em Biometria e Estatística Aplicada) - Universidade Federal Rural de Pernambuco, Recife, 2010.

BERTALANFFY, L. Von. Quantitative laws for metabolism and growth. Quaterly Review of Biology, Stony Brook, v. 32, n. 3, p. 217-231, 1957. BRITO, C. C. R. et al. Modelos de crescimento resultantes da combinação e variações dos modelos de Chapman-Richards e Silva-Bailey em Leucaena leucocephala (Lam.) de Wit. Ciência Florestal, Santa Maria, v. 17, n. 2, p. 175-185, 2007.

CLUTTER, J. L. et al. Timber management: a quantitative approach. New York: John Wiley, 1983. $333 \mathrm{p}$.

CYSNEIROS, F. J. A et al. Restricted methods in symmetrical linear regression models. Computational Statistics and Data Analysis, Maryland Heights, v. 49, n. 3, p. 689-708, 2005a.

CYSNEIROS, F. J. A Métodos Restritos e Validação de Modelos Simétricos de Regressão. 2004. 147 f. Tese (Doutorado em Estatística) - Universidade de São Paulo, São Paulo, 2004.

CYSNEIROS, F. J. A. et al. Modelos simétricos aplicados. In: ESCOLA DE MODELOS DE REGRESSÃO, 9., 2005, São Paulo. Modelos simétricos aplicados. São Paulo: ABE, 2005b. $100 \mathrm{p}$.

DAVIS, L. S.; JOHNSON, K. N. Forest management. 3th ed. New York: McGraw-Hill, 1987. $789 \mathrm{p}$.

ENCARNAÇÃO， C. R. F. da. Observações meteorológicas e tipos climáticos das unidades e campos experimentais da empresa IPA. Recife: Empresa Pernambucana de Pesquisa Agropecuária, 1980. $110 \mathrm{p}$.

GALEA, M. et al. On Diagnostic in Symmetrical 
Nonlinear Models. Statistics and Probability Letters, v. 73, n. 4, p. 459-467, 2005.

LANGE, K. L. et al.Robust statistical modeling using the $t$ distribution. Journal of the American Statistical Association, v. 48, n. 408, p. 621-625, 1989.

LIMA, W. P. Impacto ambiental do eucalipto.2. ed. São Paulo: EDUSP, 1993. 301 p.

PRODAN, M. Forest biometrics. Oxford: Pergamon Press, 1968. $447 \mathrm{p}$.

SANTOS, C. S. A. Modelos simétricos transformados não-lineares com aplicação na estimativa volumétrica em híbrido de Eucalyptus tereticornis no Polo Gesseiro do Araripe. 2010.
93 f. Dissertação (Mestrado em Biometria e Estatística Aplicada) - Universidade Federal Rural de Pernambuco, Recife, 2010.

SILVA, J. A. A. Dynamics of stand structure in fertilized slash pine plantations. 1986. 133 f. Thesis (Doctor of Philosophy) - University of Georgia, Athens, 1986.

SPATHELF, P.; NUTTO, L. Modelagem aplicada ao crescimento florestal. Santa Maria: UFSM, 2000, $70 \mathrm{p}$.

TAYLOR, J. M. G. Properties of modelling the error distribution with an extra shape parameter. Computational statistics and data analysis, v. 13, n. 1, p. 33-46, 1992. 